\title{
Comparative Analysis of Cellular and Growth Factor Composition in Bone Marrow Aspirate Concentrate and Platelet-Rich Plasma
}

\author{
Hisashi Sugaya $\mathbb{D}^{1},{ }^{1}$ Tomokazu Yoshioka ${ }^{D},{ }^{1}$ Toshiki Kato, ${ }^{2}$ \\ Yu Taniguchi $\left(D,{ }^{3}\right.$ Hiroshi Kumagai, ${ }^{3}$ Kojiro Hyodo, ${ }^{3}$ Osamu Ohneda, ${ }^{2}$ \\ Masashi Yamazaki, ${ }^{3}$ and Hajime Mishima ${ }^{3}$ \\ ${ }^{1}$ Division of Regenerative Medicine for Musculoskeletal System, Department of Orthopedics Surgery, Faculty of Medicine, \\ University of Tsukuba, 1-1-1 Tennodai, Tsukuba, Ibaraki 305-8575, Japan \\ ${ }^{2}$ Laboratory of Regenerative Medicine and Stem Cell Biology, Graduate School of Comprehensive Human Sciences, \\ University of Tsukuba, 1-1-1 Tennodai, Tsukuba, Ibaraki 305-8575, Japan \\ ${ }^{3}$ Department of Orthopedic Surgery, Faculty of Medicine, University of Tsukuba, 1-1-1 Tennodai, Tsukuba, Ibaraki 305-8575, Japan
}

Correspondence should be addressed to Hisashi Sugaya; h.sugaya.1205@tsukuba-seikei.jp

Received 13 October 2017; Revised 18 January 2018; Accepted 29 January 2018; Published 25 February 2018

Academic Editor: Luis F. Porrata

Copyright (c) 2018 Hisashi Sugaya et al. This is an open access article distributed under the Creative Commons Attribution License, which permits unrestricted use, distribution, and reproduction in any medium, provided the original work is properly cited.

\begin{abstract}
The purpose of this study was to quantify the stem cell and growth factor (GF) contents in the bone marrow aspirate concentrate (BMAC) and platelet-rich plasma (PRP) prepared from whole blood using a protocol established in our laboratory. We examined 10 patients with osteonecrosis of the femoral head who were treated by autologous BMAC transplantation at our hospital between January 2015 and June 2015. We quantified CD34+ and CD31-CD45-CD90+CD105+ cells in BMAC and PRP by flow cytometry. Additionally, we measured various GFs, that is, basic fibroblast growth factor (b-FGF), platelet-derived growth factor-BB (PDGF$\mathrm{BB}$ ), vascular endothelial growth factor (VEGF), transforming growth factor- $\beta 1$ (TGF- $\beta 1$ ), and bone morphogenetic protein-2 (BMP-2) in BMAC and PRP using enzyme-linked immunosorbent assays and statistical analyses. CD34+ and CD31-45-90+105+ cells accounted for approximately $1.9 \%$ and $0.03 \%$ of cells in BMAC and no cells in PRP. The concentration of b-FGF was higher in BMAC than in PRP $(P<0.001)$, whereas no significant differences in the levels of PDGF-BB, VEGF, TGF- $\beta 1$, and BMP-2 were observed between the two types of sample. BMAC had an average of $1.9 \%$ CD34+ and $0.03 \%$ CD31-45-90+105+ cells and higher levels of b-FGF than those of PRP.
\end{abstract}

\section{Introduction}

Bone marrow aspirate (BMA) is a mixture of aspirated BM cells, small tissue fragments, and venous sinus blood (peripheral blood). Its bone-stimulating effects were first described in experiments using rabbits [1]; it was later discovered that fibroblast-like cells with adhesive, colony-forming, and osteogenic potential are present in BM [2]. It is now known that mesenchymal stem cells in the BM have the potential to differentiate into various mesenchymal tissues [3]. BMAs have been used in orthopedic surgery for nonunion [4], the treatment of osteonecrosis (especially femoral head necrosis) and osseous defects, and bone lengthening [5-8].
We previously developed a treatment for osteonecrosis and nonunion using 30-40 $\mathrm{ml}$ of BMA concentrate (BMAC) produced without special instruments from $300-400 \mathrm{ml}$ of BMAs harvested from the ilium and centrifuged [9-14]. To ensure that the buffy coat layer of the BMA was extracted without leakage, the areas immediately above and below this layer were also manually extracted. Thus, the BMAC also contained the platelet-rich plasma (PRP) formed just above the buffy coat layer. This fraction, which is a plasma fraction containing an abundance of platelets, has clinical applications in the field of orthopedic surgery as a regenerative treatment, primarily for sports injuries $[15,16]$. Our earlier studies demonstrated the therapeutic value of PRP obtained 
from whole peripheral blood (WB) $[16,17]$. Transplanting BMAC and PRP together also has positive results [18, 19]. However, BMAC and PRP preparation methods vary from one experiment to the next; as such, there are no conclusive results regarding differences between cellular components and humoral factors contained in BMAC or PRP.

To address this issue, we analyzed the stem cell and growth factor contents of the BMAC produced using our protocol. Specifically, we quantified hematopoietic progenitor/stem cell and mesenchymal stromal/stem cell populations as well as growth factor concentrations. We also evaluated differences in the compositions of BMAC and PRP prepared from WB.

\section{Materials and Methods}

All procedures were approved by the Institutional Ethics Review Committee of the University of Tsukuba. Informed consent was obtained preoperatively from all study participants.

2.1. Patients. Ten patients with osteonecrosis of the femoral head $(\mathrm{ONFH})$ who were treated by concentrated autologous BMA transplantation at our hospital between January 2015 and June 2015 were included. The mean age of the eight male patients and two female patients was 40 years (range: $28-59$ years). In this cohort, ONFH developed in eight patients following long-term corticosteroid use and in two patients who abused alcohol.

2.2. BM Aspiration and Concentration. BM was aspirated and concentrated according to a previously published method [9-14]. Briefly, patients were given general anesthesia and $\mathrm{BM}$ was aspirated from both anterior iliac crests using a BM harvest needle (Medical Device Technologies, Gainesville, FL, USA) with $20 \mathrm{ml}$ syringes prefilled with $1.5 \mathrm{ml}$ of the anticoagulant citrate dextrose. Aspirates were collected using a BM collection kit (Baxter, Deerfield, IL, USA) and then transferred to a quadruple blood bag (Terumo, Tokyo, Japan) for concentration by a two-step centrifugation method (KUBOTA 9800; Kubota, Tokyo, Japan). The blood bag contents were first centrifuged at $1200 \times \mathrm{g}$ for $10 \mathrm{~min}$ at room temperature, and erythrocytes were removed from the original bag. The remaining contents were centrifuged at $3870 \times \mathrm{g}$ for $7 \mathrm{~min}$ at room temperature, after which the plasma and anticoagulants were removed followed by extraction of the BMAC containing the buffy coat. This technique reduced the $400 \mathrm{ml}$ volume of BMA to $30-40 \mathrm{ml}$ of BMAC, of which $4 \mathrm{ml}$ was used for experiments.

2.3. Preparation of PRP Derived from $W B$. At the time of $B M$ aspiration and concentration, $18 \mathrm{ml}$ of $\mathrm{WB}$ was obtained from the same patient. PRP was prepared from WB as previously described [20]. A 21-gauge butterfly needle was used to draw $18 \mathrm{ml}$ of $\mathrm{WB}$ from the cubital vein of the subjects, while avoiding hemolysis. The blood was collected in two $9 \mathrm{ml}$ Spitz tubes containing $3.8 \%$ citric acid and was centrifuged at $580 \times \mathrm{g}$ for $8 \mathrm{~min}$ at room temperature (PRGF-Endoret IV System; BTI Biotechnology Institute, Vitoria, Spain). The contents of each tube were separated into the erythrocyte, buffy coat, and plasma layers. In the separated plasma layer, a safety area was delineated in the upper area of the buffy coat, while avoiding aspirating the buffy coat; the upper and lower halves of the plasma layer were defined as platelet-poor plasma and PRP, respectively. About $2 \mathrm{ml}$ of PRP per Spitz tube was collected by aspiration using a dedicated aspirator (PRGF-Endoret IV; BTI Biotechnology Institute) for a total of about $4 \mathrm{ml}$ of PRP from the two tubes.

2.4. Hematological Analysis. Nucleated white blood cells and platelets in BMA, BMAC, WB, and PRP were counted using an automated system (Sysmex KX-21N; Sysmex Corp., Kobe, Japan).

2.5. Fibroblastic Colony-Forming Unit (CFU-F) Assay and Cell Characterization. To determine the presence and proportion of progenitor cells in BMA and BMAC, samples from each patient were evaluated with the CFU-F assay, as previously described $[10,12,14]$. The samples $(100 \mu \mathrm{l})$ were washed twice with phosphate-buffered saline and then resuspended in $3 \mathrm{ml}$ of growth medium composed of Dulbecco's Modified Eagle's Medium (Sigma-Aldrich, St. Louis, MO, USA) supplemented with $10 \%$ fetal bovine serum (Gibco, Grand Island, NY, USA) and antibiotic-antimycotic solution (Gibco). The cells were seeded in $60 \mathrm{~cm}^{2}$ dishes and cultured at $37^{\circ} \mathrm{C}$ in a humidified atmosphere of $5 \% \mathrm{CO}_{2}$. The medium was replaced 2 days later and nonadherent cells were removed; thereafter, the medium was replaced twice a week. After 2 weeks, the medium was removed and cells were stained with $0.5 \%$ Crystal Violet (Sigma-Aldrich) in methanol for $5 \mathrm{~min}$ and washed twice with distilled water before the number of CFUFs was counted. Colonies of $<2 \mathrm{~mm}$ in diameter and those that were only faintly stained were excluded. The number of progenitor cells is expressed as the number of CFU-Fs per $10^{6}$ nucleated cells.

In order to analyze the cellular composition of BMAC, the number of CD34+ cells, which are precursors of hematopoietic cells, and CD31-45-90+105+ cells were determined. $\mathrm{BM}$ cells in BMAC were incubated in red blood cell lysis buffer (VersaLyse; Beckman Coulter, Brea, CA, USA) at $37^{\circ} \mathrm{C}$ for $5 \mathrm{~min}$, followed by incubation for $30 \mathrm{~min}$ at $4^{\circ} \mathrm{C}$ with fluorescein isothiocyanate-conjugated anticluster of differentiation (CD)31 (1:40), phycoerythrin (PE)/Cy5-conjugated anti-CD90 (1:200), energy-coupled dye (PE-Texas Red)conjugated anti-CD45 (1:40), and allophycocyanin- (APC-) conjugated anti-CD105 $(1: 200)$ antibodies (all from BD Pharmingen, San Jose, CA, USA), and APC-conjugated antiCD34 antibody (1:200; Biolegend, San Diego, CA, USA). Matching isotype control antibodies were used as a control. After incubation, labeled cells were sorted on a Gallios flow cytometer (Beckman Coulter), and data were analyzed using Kaluza software (Beckman Coulter).

2.6. Growth Factor (GF) Quantification. The BMAC and PRP were incubated at $37^{\circ} \mathrm{C}$ for $1 \mathrm{~h}$ after adding $5 \%$ calcium chloride (BTI Biotechnology Institute) and centrifuged at $1000 \times \mathrm{g}$ for $20 \mathrm{~min}$ at $4^{\circ} \mathrm{C}$. The supernatant was removed and stored at $-80^{\circ} \mathrm{C}$. The cryopreserved supernatant was 
thawed at room temperature before use. The concentrations of basic fibroblast growth factor (b-FGF), platelet-derived growth factor- (PDGF-) BB, transforming growth factor(TGF-) $\beta 1$, vascular endothelial growth factor (VEGF), and bone morphogenetic protein- (BMP-) 2 were measured using enzyme-linked immunosorbent assay kits specific for each GF (R\&D Systems, Minneapolis, MN, USA) according to the manufacturer's recommendations. All standards and samples were analyzed in duplicate.

2.7. Statistical Analysis. The numbers of nucleated cells and platelets in BMAC and PRP before and after the concentration procedure were analyzed using Wilcoxon's rank sum tests. The GF quantification results for BMAC and PRP were analyzed using Mann-Whitney $U$ tests. $P<0.05$ was considered statistically significant. Analyses were performed using IBM SPSS Statistics v.24.0 (IBM Corp., Armonk, NY, USA).

\section{Results}

3.1. Characterization of Cells in BMAC and PRP. The volume of BMA obtained was $402 \pm 35 \mathrm{ml}$; after concentration, the volume was $35.4 \pm 1.6 \mathrm{ml}$. The average ratio of the volumes after and before concentration was 11.4. The findings of the hematological analysis are presented in Figure 1. On average, the number of nucleated cells was 5.53-fold higher in BMAC $\left(43.0 \pm 15.1 \times 10^{3} / \mu \mathrm{l}\right)$ than in WB $\left(7.78 \pm 3.06 \times 10^{3} / \mu \mathrm{l}\right)$, and BMAC had higher levels of WBC than BMA $(P<0.001)$. The average platelet count was also 5.93-fold higher in BMAC $\left(38.1 \pm 21.1 \times 10^{4} / \mu \mathrm{l}\right)$ than in BMA $\left(6.42 \pm 3.06 \times 10^{4} / \mu \mathrm{l}\right)$ and BMAC had higher levels of PLT than BMA $(P<0.001)$. No nucleated cells were detected in PRP samples. The average platelet count was 1.49-fold higher in PRP $(28.8 \pm 11.6 \times$ $\left.10^{4} / \mu \mathrm{l}\right)$ than in WB $\left(19.3 \pm 4.04 \times 10^{4} / \mu \mathrm{l}\right)$ and PRP had higher levels of PLT than WB $(P=0.049)$. Based on platelet counts and the presence of white blood cells and neutrophils, the PRP preparation was classified as $\mathrm{P} 2-\mathrm{x}-\mathrm{B} \beta$ according to the platelet-activation white blood cell classification system [21].

The mean CFU-F count in BMAC $(196 \pm 161 / \mathrm{ml})$ was higher than that in BMA $(31.6 \pm 37.0 / \mathrm{ml})$. The mean concentration rate of CFU-F was $6.0 \pm 4.4$, and the number of CFU-F per $10^{6}$ nucleated cells in BMAC was $4.62 \pm 4.07$. The mean percentage of CD34+ cells per total BM cells was $1.87 \%$ (range: $0.60 \%-4.25 \%$ ), and the mean percentage of CD31-45-90+105+ cells per total BM cells was $0.030 \%$ (range: $0.001 \%-0.101 \%$ ) (Figure 2).

3.2. GF Levels in BMAC and PRP. GF levels in BMAC were as follows: b-FGF, $6.78 \pm 5.87 \times 10^{1} \mathrm{pg} / \mathrm{ml}$; PDGF-BB, $5.28 \pm$ $2.57 \times 10^{3} \mathrm{pg} / \mathrm{ml}$; VEGF, $1.76 \pm 1.18 \times 10^{2} \mathrm{pg} / \mathrm{ml}$; TGF- $\beta 1$, $1.56 \pm 1.33 \times 10^{4} \mathrm{pg} / \mathrm{ml}$; and BMP-2, $9.99 \pm 0.59 \times 10^{1} \mathrm{pg} / \mathrm{ml}$. The levels in PRP were as follows: b-FGF, $1.07 \pm 0.35 \times$ $10^{1} \mathrm{pg} / \mathrm{ml}$; PDGF-BB, $6.71 \pm 4.21 \times 10^{3} \mathrm{pg} / \mathrm{ml}$; VEGF, $2.39 \pm$ $2.11 \times 10^{2} \mathrm{pg} / \mathrm{ml}$; TGF- $\beta 1,2.65 \pm 1.46 \times 10^{4} \mathrm{pg} / \mathrm{ml}$; and BMP$2,9.87 \pm 0.50 \times 10^{1} \mathrm{pg} / \mathrm{ml}$. The concentration of b-FGF was higher in BMAC than in PRP $(P<0.001)$, whereas no significant differences in the levels of the other GFs were observed between the two types of sample (Figure 3). GF levels for each patient are presented in Figure 4.

\section{Discussion}

CD34+ and CD31-45-90+105+ cells accounted for approximately $1.9 \%$ and $0.03 \%$ of cells in BMAC, respectively. BMAC contained a higher concentration of b-FGF than PRP, although the two types of sample had similar concentrations of the other GFs. It was previously demonstrated that CD34+ positive cells make up about $1 \%$ of the total number of mononuclear cells [22]; other investigators have reported that the proportion of nucleated cells in BMAC that were CD34+ was $1.0 \% \pm 0.2 \%$ [23], which is consistent with our observations.

CFU-Fs constitute $0.01 \%-0.001 \%$ of all BM mononuclear cells [24], corresponding to a mesenchymal stem cell (MSC) ratio of $1 / 100,000$ hematopoietic cells [25]. Although the average number of CD31-45-90+105+ cells was relatively high $(0.03 \%)$, the average number of CFU-Fs was small $\left(4 / 10^{6}\right.$ mononuclear cells). Other investigators have obtained results similar to those of the present study using the same BMAC preparation method, with an average number of CFU-Fs of $2.55 / 10^{6}$ mononuclear cells [14]. On the other hand, a CD45-CD271+ cell fraction of $0.016 \%$ (from $0.009 \%-0.032 \%$ ) was observed in BMAs, with 1520 cells per $1 \mathrm{ml}$ of BM $(96-20,992)$ and a significantly lower average CFU-F count, that is, 60 (3-900) per $1 \mathrm{ml}$ of BM [26] or comparable results $[27,28]$. In the present study, we also obtained a high cell count based on an analysis of cell surface antigen expression as compared to CFU-F.

To measure GF levels in BMAC and PRP, 5\% calcium chloride was added to both samples, that is, BMAC and PRP, for platelet activation. Sánchez et al. reported that calcium chloride leads to platelet activation and the hydrolysis of prothrombin into thrombin, which simultaneously causes the release of myriad growth factors and the polymerization of fibrin [20].

A comparison of GF levels in BMAC and WB PRP revealed no significant differences in PDGF, TGF- $\beta 1$, interleukin- (IL-) 8 or interleukin-6, tumor necrosis factor- $\alpha$, interferon- $\gamma$, or FGF-1 levels; however, VEGF and IL-1 receptor antagonist levels were higher in BMAC than in PRP [29]. We found similar results for PDGF and TGF- $\beta 1$ but observed no significant differences in VEGF expression, whereas b-FGF was elevated in BMAC as compared to PRP.

VEGF modulates angiogenesis, migration, and mitosis of endothelial cells as well as the creation of the blood vessel lumen $[30,31]$. The PRP VEGF concentration of $2.39 \pm$ $2.11 \times 10^{2} \mathrm{pg} / \mathrm{ml}$ obtained here is comparable to the value of $2.76 \pm 2.73 \times 10^{2} \mathrm{pg} / \mathrm{ml}$ found in a previous study [29]. However, others have reported VEGF concentrations in PRP of $76-854 \mathrm{pg} / \mathrm{ml}$ [30] and $10 \pm 12 \mathrm{pg} / \mathrm{ml}$ [29]. Moreover, we found a BMAC VEGF concentration of $1.76 \pm 1.18 \times$ $10^{2} \mathrm{pg} / \mathrm{ml}$, which is higher than that reported by Cassano et al. [29], but comparable to the results of Alsousou et al. [30] (Table 1). Our data suggest that PRP and BMAC contain equivalent amounts of VEGF. However, given that this is 


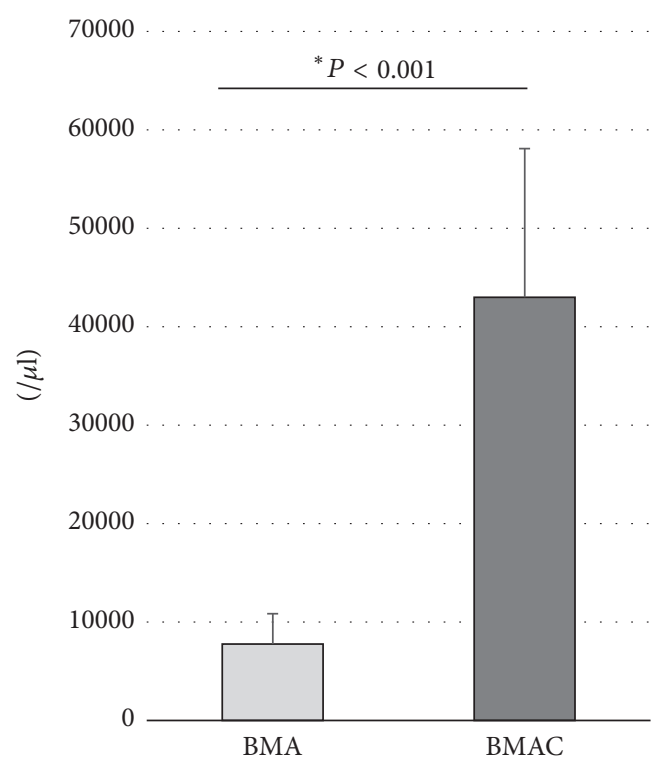

(a)

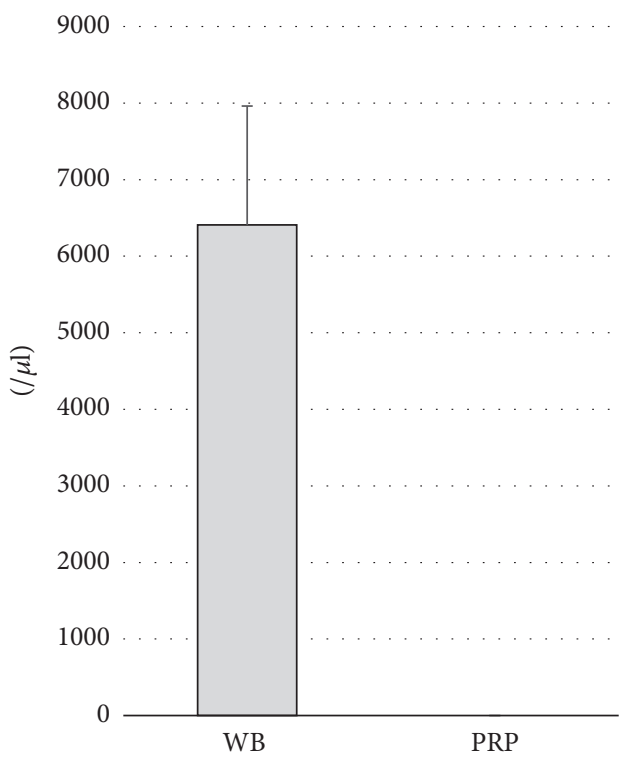

(c)

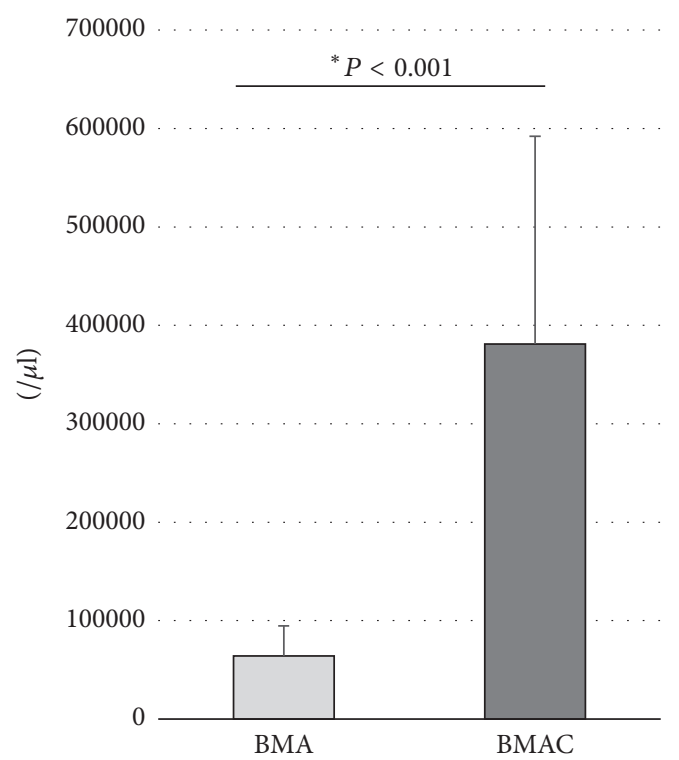

(b)

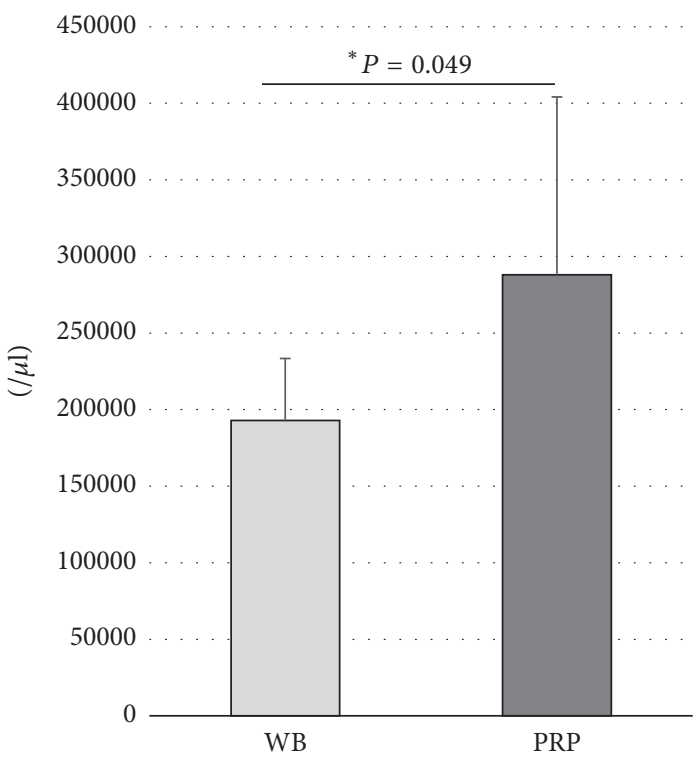

(d)

FIGURE 1: Numbers of nucleated cells and platelets in BMAC and PRP before and after concentration. (a) BMAC had higher levels of WBC than BMA $(P<0.001)$. (b) BMAC had higher levels of PLT than BMA $(P<0.001)$. (c) No nucleated cells were detected in PRP samples. $(\mathrm{d})$ PRP had higher levels of PLT than WB $(P=0.049) .\left({ }^{*} P<0.05\right)$.

contradicted by other reports, a more detailed analysis of a larger number of cases is warranted.

FGF belongs to a family of heparin-binding polypeptide GFs that includes b-FGF (FGF-2), which activates signaling pathways involved in the development and maintenance of cartilage [32]. b-FGF is known to induce cell proliferation and chondrogenic differentiation in human BM MSCs [33]. However, there are no studies of b-FGF concentrations in BMAC; in one study, FGF-1 was not detected in BMAC or PRP [29], but b-FGF was not evaluated. We found that bFGF was present at higher concentrations in BMAC than in PRP.
b-FGF is involved in the maintenance of the MSC cytoplasmic volume and spindle morphology [34-36]. MSCs cultured with FGF maintained the spindle shape typical of fibroblasts, whereas, in the absence of FGF, cells developed a large cytoplasmic volume [34]. Identical results were obtained for MSCs derived from human BMAs [35-37]. In this study, we found MSCs in BMAC, but not in PRP, and observed higher concentrations of b-FGF in BMAC; however, it remains to be determined whether b-FGF plays a role in maintaining MSC cell morphology.

Using our method, leukocytes were not detected in PRP, and the platelet counts in PRP were lower than those for other 


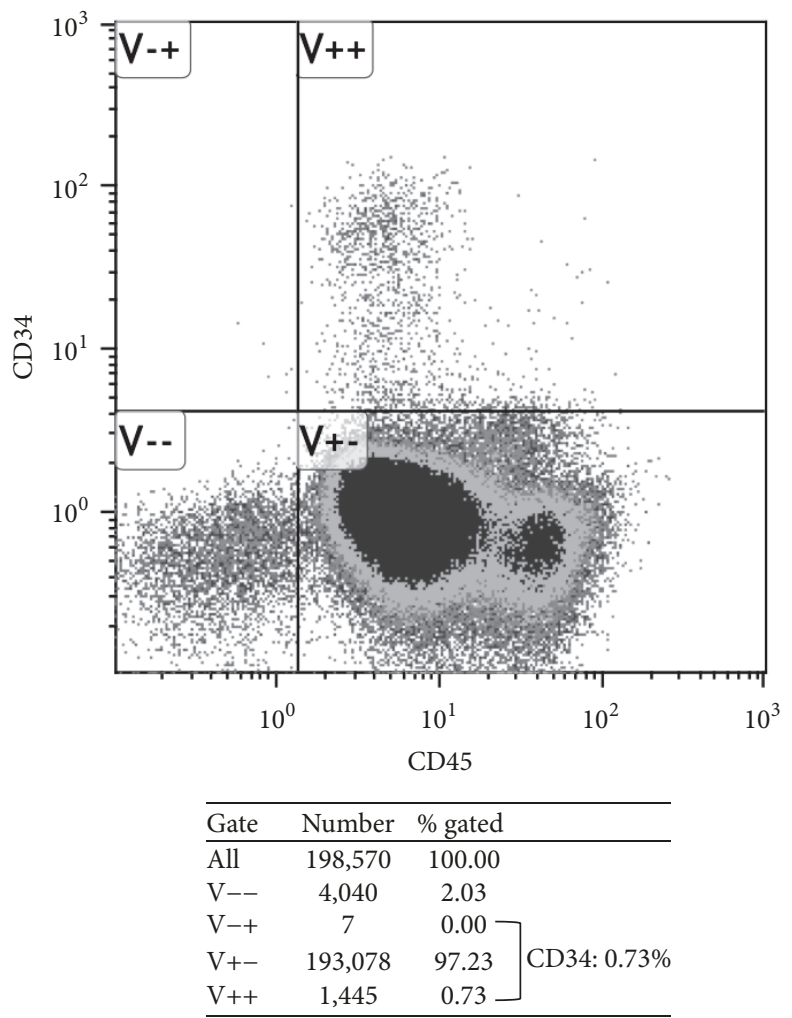

(a)
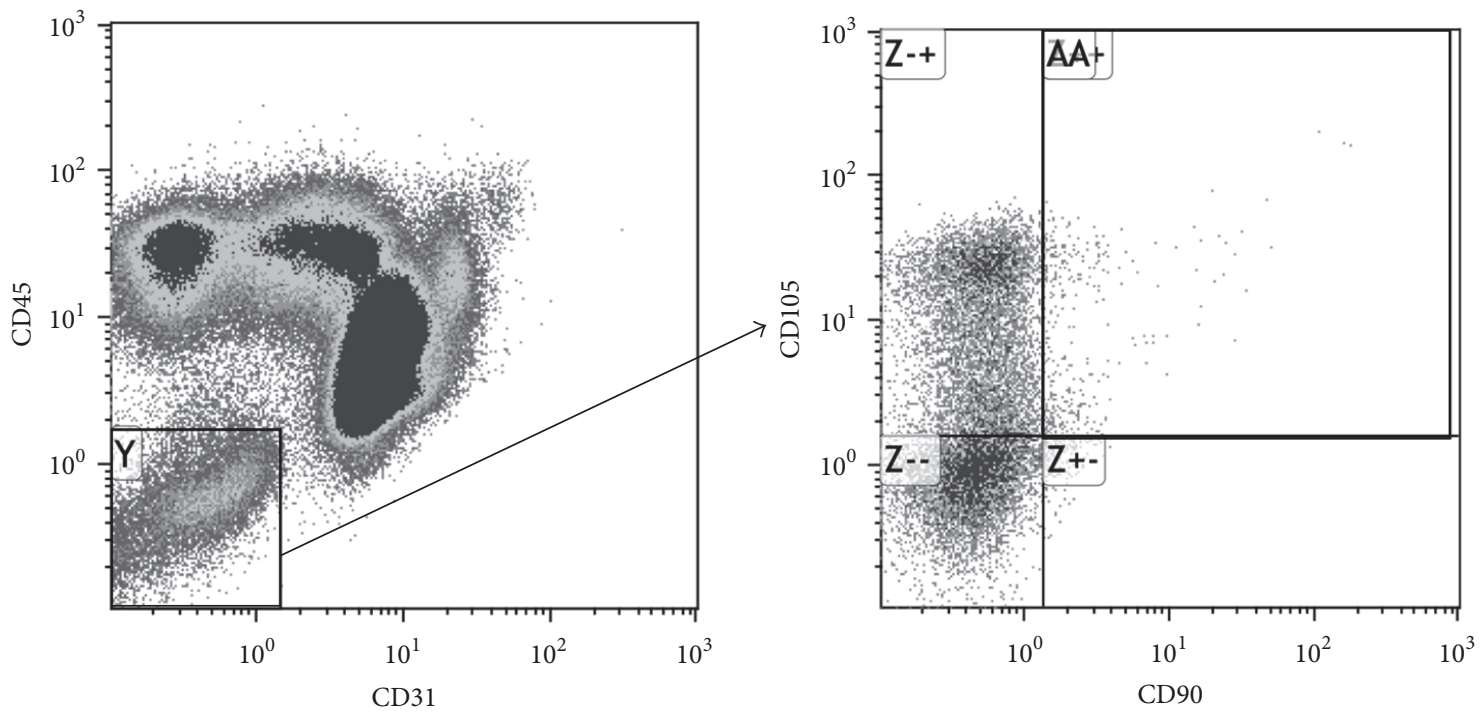

\begin{tabular}{lcc}
\hline Gate & Number & \% gated \\
\hline All & 726,569 & 100.00 \\
Y & 16,062 & 2.21 \\
\hline
\end{tabular}

\begin{tabular}{lcc}
\hline Gate & Number & \% gated \\
\hline All & 16,062 & 100.00 \\
AA & 381 & 2.37 \\
\hline
\end{tabular}

(b)

FIGURE 2: Flow cytometry results for CD34+ cells and CD31-45-90+105+ cells. Results for a 37-year-old woman with corticosteroid-induced ONFH. (a) The percentage of CD34+ cells per total BM cells was $0.73 \%(1,452 / 198,570)$. (b) The percentage of CD31-45-90+105+ cells per total BM cells was $0.052 \%(381 / 726,569)$. 


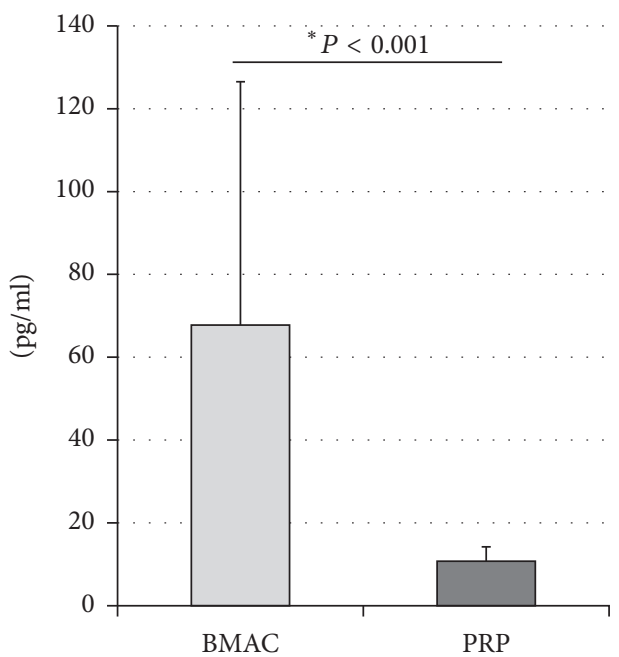

(a) b-FGF

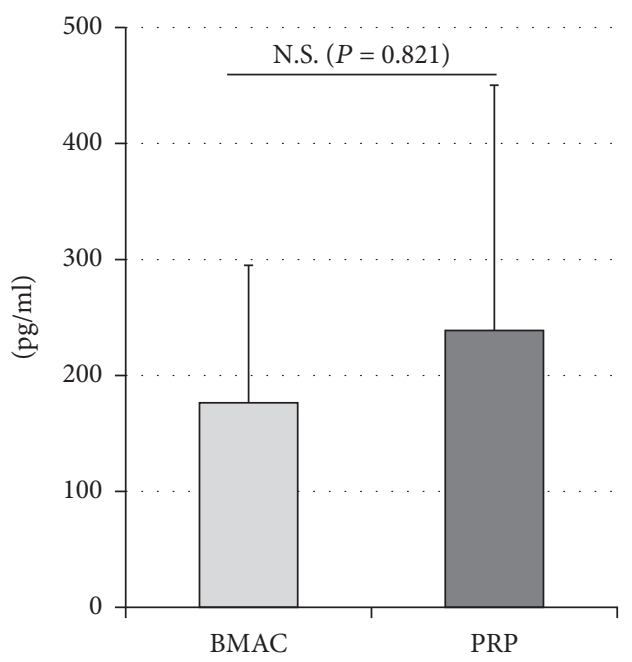

(c) VEGF

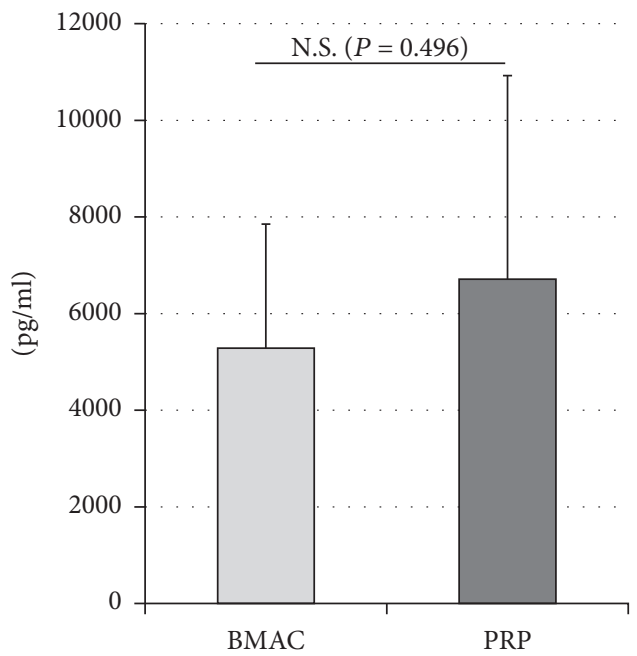

(b) PDGF-BB

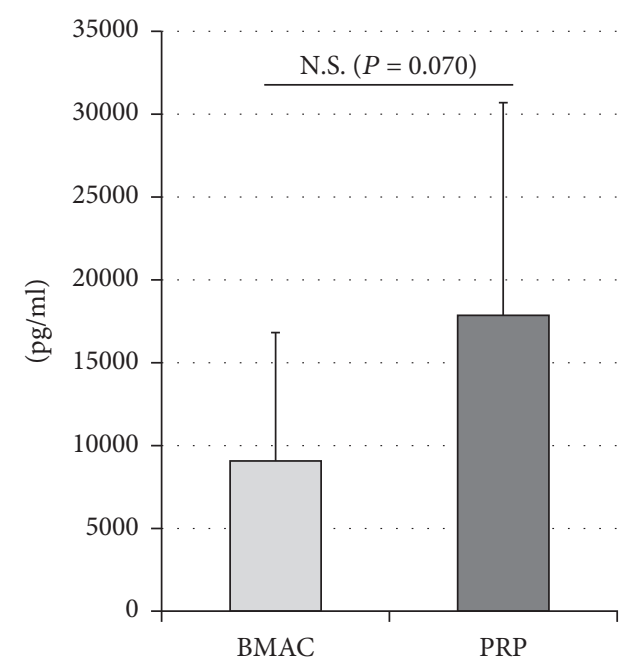

(d) TGF- $\beta 1$

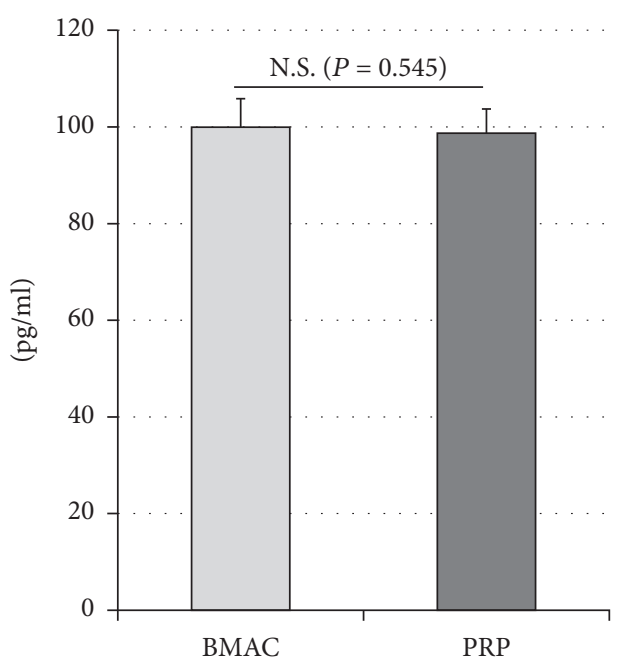

(e) BMP-2

FIGURE 3: GF levels in BMAC and PRP. (a) BMAC had higher levels of b-FGF than PRP $(P<0.001)$. (b-e) There were no differences between PRP and BMAC in terms of PDGF-BB, VEGF, TGF- $\beta 1$, and BMP-2 levels. $\left({ }^{*} P<0.05\right)$. 


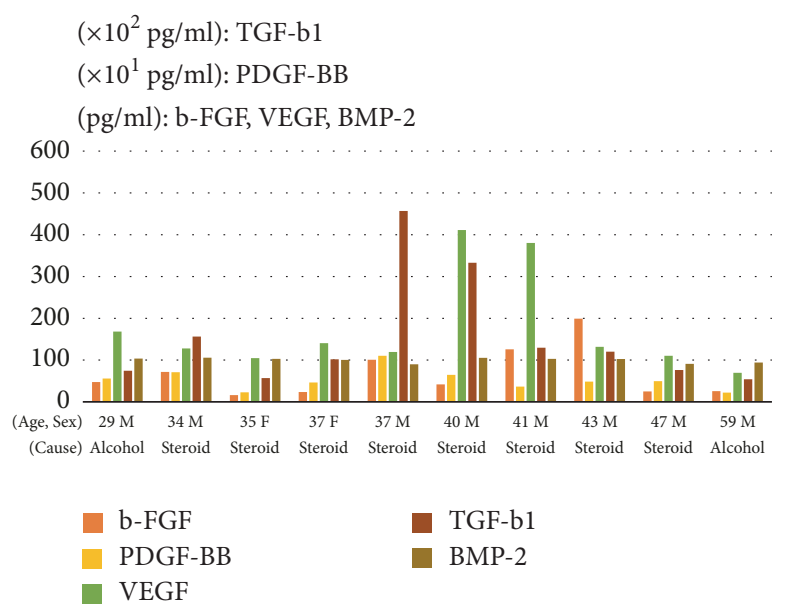

(a)

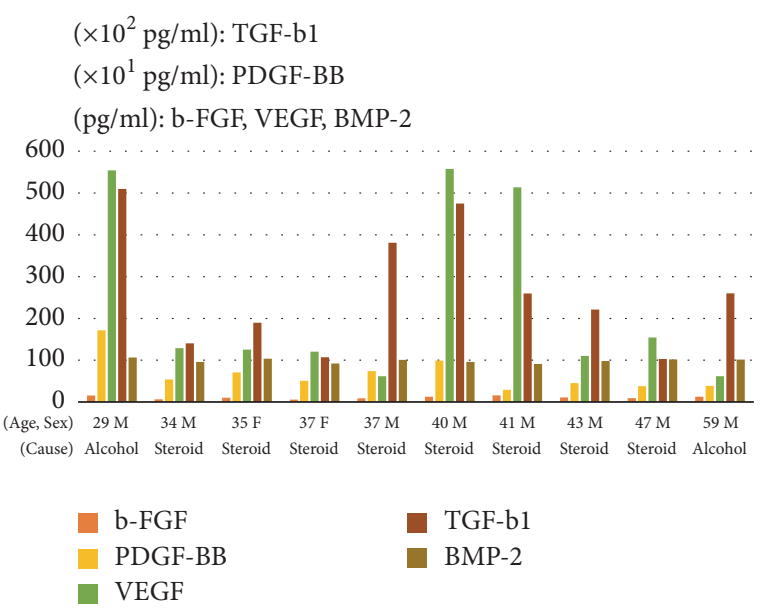

(b)

FIgURE 4: GF levels for each patient. (a) BMAC; (b) PRP.

TABLE 1: Comparison of VEGF levels in BMAC and PRP.

\begin{tabular}{lcc}
\hline & \multicolumn{2}{c}{ VEGF level (pg/ml) } \\
& BMAC & PRP \\
\hline Our study & $239 \pm 211$ & $176 \pm 118$ \\
Cassano et al. [29] & $276 \pm 273$ & $10 \pm 12$ \\
Alsousou et al. [30] & - & 76 to 854 \\
\hline
\end{tabular}

methods. Some studies have reported platelet or leukocyte counts in PRP and GF levels. In PRP prepared using five different systems, Magalon et al. found positive correlations between platelet counts in PRP and levels of VEGF, PDGF$\mathrm{AB}$, and TGF- $\beta 1$ and between leukocyte counts in PRP and levels of VEGF [38]. Zimmermann et al. reported remarkable interindividual variation in GF levels and no correlation between GF levels and platelet concentration [39]. Castillo et al. reported a positive correlation between GF levels and leukocyte counts in PRP [40]. Our method might have a disadvantage with respect to GF levels in PRP owing to the relatively low enrichment for platelets.

These findings are applicable to the procedures and instruments that we developed to obtain BMAC and PRP; similar tests, including comparative analyses, should be performed when other methods are used for sample preparation. One limitation of this study was the small sample size. For patients with ONFH, blood sampling to obtain PRP was not directly related to their treatment and accordingly the number of samples was limited. Furthermore, since BMAC and PRP preparation methods can vary, caution must be exercised when comparing the results of different studies.

\section{Conclusion}

We produced BMAC that contained an average of $1.9 \%$ CD34+ and $0.03 \%$ CD31-45-90+105+ cells. BMAC had higher levels of b-FGF than PRP, although the two samples had comparable levels of PDGF-BB, VEGF, TGF- $\beta 1$, and
BMP-2. These findings suggest that our protocol can be used to produce BMAC for therapeutic applications, such as the treatment of osteonecrosis and nonunion.

\section{Conflicts of Interest}

The authors declare that they have no conflicts of interest.

\section{Authors' Contributions}

Hisashi Sugaya and Tomokazu Yoshioka contributed equally to this work.

\section{Acknowledgments}

The authors thank Chizuko Fujisawa (Laboratory of Regenerative Medicine and Stem Cell Biology, Graduate School of Comprehensive Human Sciences, University of Tsukuba) for technical assistance regarding analysis for characterization of cells and Yohei Tomaru, M.D. (Department of Orthopedic Surgery, Faculty of Medicine, University of Tsukuba), for technical advice regarding statistical analysis.

\section{References}

[1] E. Goujon, "Recherches experimentales sur les proprieties physiologiques de la moelle des os," Journal of Anatomy and Physiology, vol. 6, pp. 399-412, 1869.

[2] A. J. Friedenstein, "Stromal mechanisms of bone marrow: cloning in vitro and retransplantation in vivo.," Hämatologie und Bluttransfusion, vol. 25, pp. 19-29, 1980.

[3] A. I. Caplan, "Mesenchymal stem cells," Journal of Orthopaedic Research, vol. 9, no. 5, pp. 641-650, 1991.

[4] J. F. Connolly and R. Shindell, "Percutaneous marrow injection for an ununited tibia," The Nebraska medical journal, vol. 71, no. 4, pp. 105-107, 1986.

[5] M. A. Imam, J. Holton, L. Ernstbrunner et al., "A systematic review of the clinical applications and complications of bone marrow aspirate concentrate in management of bone defects 
and nonunions," International Orthopaedics, vol. 41, no. 11, pp. 2213-2220, 2017.

[6] F. Salamanna, D. Contartese, N. Nicoli Aldini et al., "Bone marrow aspirate clot: A technical complication or a smart approach for musculoskeletal tissue regeneration?" Journal of Cell Physiology, vol. 233, pp. 2723-2732, 2018.

[7] N. S. Piuzzi, J. Chahla, J. B. Schrock et al., "Evidence for the use of cell-based therapy for the treatment of osteonecrosis of the femoral head: A systematic review of the literature," The Journal of Arthroplasty, vol. 32, no. 5, pp. 1698-1708, 2017.

[8] C. Papakostidis, T. H. Tosounidis, E. Jones, and P. V. Giannoudis, "The role of "cell therapy" in osteonecrosis of the femoral head: a systematic review of the literature and metaanalysis of 7 studies," Acta Orthopaedica, vol. 87, no. 1, pp. 72-78, 2016.

[9] Y. Tomaru, T. Yoshioka, H. Sugaya et al., "Hip preserving surgery with concentrated autologous bone marrow aspirate transplantation for the treatment of asymptomatic osteonecrosis of the femoral head: Retrospective review of clinical and radiological outcomes at 6 years postoperatively," BMC Musculoskeletal Disorders, vol. 18, no. 1, article no. 292, 2017.

[10] H. Mishima, H. Sugaya, T. Yoshioka et al., "The safety and efficacy of combined autologous concentrated bone marrow grafting and low-intensity pulsed ultrasound in the treatment of osteonecrosis of the femoral head," European Journal of Orthopaedic Surgery and Traumatology, vol. 26, no. 3, pp. 293298, 2016.

[11] H. Sugaya, H. Mishima, K. Aoto et al., "Percutaneous autologous concentrated bone marrow grafting in the treatment for nonunion," European Journal of Orthopaedic Surgery and Traumatology, vol. 24, no. 5, pp. 671-678, 2014.

[12] T. Yoshioka, H. Mishima, H. Akaogi, S. Sakai, M. Li, and N. Ochiai, "Concentrated autologous bone marrow aspirate transplantation treatment for corticosteroid-induced osteonecrosis of the femoral head in systemic lupus erythematosus," International Orthopaedics, vol. 35, no. 6, pp. 823-829, 2011.

[13] K. Hyodo, T. Yoshioka, H. Sugaya et al., "Predicting risk factors of total hip arthroplasty conversion after concentrated autologous bone marrow aspirate transplantation for the treatment of idiopathic osteonecrosis of the femoral head: a retrospective review of 213 hips at a mean follow-up of 5 years," The Journal of Hip Surgery, vol. 01, pp. 10-1055, 2017.

[14] S. Sakai, H. Mishima, T. Ishii et al., "Concentration of bone marrow aspirate for osteogenic repair using simple centrifugal methods," Acta Orthopaedica, vol. 79, no. 3, pp. 445-448, 2008.

[15] R. E. Marx, "Platelet-rich plasma (PRP): what is PRP and what is not PRP?" Implant Dentistry, vol. 10, no. 4, pp. 225-228, 2001.

[16] T. Yoshioka, A. Kanamori, T. Washio et al., "The effects of plasma rich in growth factors (PRGF-Endoret) on healing of medial collateral ligament of the knee," Knee Surgery, Sports Traumatology, Arthroscopy, vol. 21, no. 8, pp. 1763-1769, 2013.

[17] K. Aoto, A. Kanamori, T. Yoshioka, K. Uemura, M. Sakane, and M. Yamazaki, "Circadian variation of growth factor levels in platelet-rich plasma," Clinical Journal of Sport Medicine, vol. 24, no. 6, pp. 509-512, 2014.

[18] H. Kitoh, T. Kitakoji, H. Tsuchiya et al., "Transplantation of marrow-derived mesenchymal stem cells and platelet-rich plasma during distraction osteogenesis - A preliminary result of three cases," Bone, vol. 35, no. 4, pp. 892-898, 2004.

[19] D. H. Lee, K. J. Ryu, J. W. Kim, K. C. Kang, and Y. R. Choi, "Bone Marrow Aspirate Concentrate and Platelet-rich Plasma
Enhanced Bone Healing in Distraction Osteogenesis of the Tibia," Clinical Orthopaedics and Related Research, vol. 472, no. 12, pp. 3789-3797, 2014.

[20] M. Sánchez, E. Anitua, D. Delgado, P. Sánchez, G. Orive, and S. Padilla, "Muscle repair: Platelet-rich plasma derivates as a bridge from spontaneity to intervention," Injury, vol. 45, no. 4, pp. S7-S14, 2014.

[21] J. M. Delong, R. P. Russell, and A. D. Mazzocca, "Plateletrich plasma: the PAW classification system," The Journal of Arthroscopic and Related Surgery, vol. 28, no. 7, pp. 998-1009, 2012.

[22] C. I. Civin, T. Trischmann, N. S. Kadan et al., "Highly purified CD34-positive cells reconstitute hematopoiesis," Journal of Clinical Oncology, vol. 14, no. 8, pp. 2224-2233, 1996.

[23] V. Gangji, J. P. Hauzeur, C. Matos, V. de Maertelaer, M. Toungouz, and M. Lambermont, “Treatment of osteonecrosis of the femoral head with implantation of autologous bone-marrow cells: a pilot study," The Journal of Bone \& Joint Surgery, vol. 86, no. 6, pp. 1153-1160, 2004.

[24] H. Castro-Malaspina, R. E. Gay, G. Resnick et al., "Characterization of human bone marrow fibroblast colony-forming cells (CFU-F) and their progeny," Blood, vol. 56, no. 2, pp. 289-301, 1980.

[25] E. Jones and D. McGonagle, "Human bone marrow mesenchymal stem cells in vivo," Rheumatology, vol. 47, no. 2, pp. 126-131, 2008.

[26] J. J. El-Jawhari, R. Cuthbert, D. McGonagle, E. Jones, and P. V. Giannoudis, "The CD45lowCD271high Cell Prevalence in Bone Marrow Samples May Provide a Useful Measurement of the Bone Marrow Quality for Cartilage and Bone Regenerative Therapy," Journal of Bone and Joint Surgery - American Volume, vol. 99, no. 15, pp. 1305-1313, 2017.

[27] R. Cuthbert, S. A. Boxall, H. B. Tan, P. V. Giannoudis, D. Mcgonagle, and E. Jones, "Single-platform quality control assay to quantify multipotential stromal cells in bone marrow aspirates prior to bulk manufacture or direct therapeutic use," Cytotherapy, vol. 14, no. 4, pp. 431-440, 2012.

[28] A. Tormin, O. Li, J. C. Brune et al., "CD146 expression on primary nonhematopoietic bone marrow stem cells is correlated with in situ localization," Blood, vol. 117, no. 19, pp. 5067-5077, 2011.

[29] J. M. Cassano, J. G. Kennedy, K. A. Ross, E. J. Fraser, M. B. Goodale, and L. A. Fortier, "Bone marrow concentrate and platelet-rich plasma differ in cell distribution and interleukin 1 receptor antagonist protein concentration," Knee Surgery, Sports Traumatology, Arthroscopy, vol. 21, pp. 333-342, 2018.

[30] J. Alsousou, M. Thompson, P. Hulley, A. Noble, and K. Willett, "The biology of platelet-rich plasma and its application in trauma and orthopaedic surgery: A review of the literature," The Journal of Bone \& Joint Surgery (British Volume), vol. 91, no. 8, pp. 987-996, 2009.

[31] N. Ferrara and H.-P. Gerber, "The role of vascular endothelial growth factor in angiogenesis," Acta Haematologica, vol. 106, no. 4, pp. 148-156, 2001.

[32] D. M. Ornitz and P. J. Marie, "FGF signaling pathways in endochondral and intramembranous bone development and human genetic disease," Genes \& Development, vol. 16, no. 12, pp. 1446-1465, 2002.

[33] M. Dominici, K. Le Blanc, I. Mueller et al., "Minimal criteria for defining multipotent mesenchymal stromal cells. The International Society for Cellular Therapy position statement," Cytotherapy, vol. 8, no. 4, pp. 315-317, 2006. 
[34] P. V. Guillot, C. Gotherstrom, J. Chan, H. Kurata, and N. M. Fisk, "Human first-trimester fetal MSC express pluripotency markers and grow faster and have longer telomeres than adult MSC," Stem Cells, vol. 25, no. 3, pp. 646-654, 2007.

[35] A. D. Murdoch, L. M. Grady, M. P. Ablett, T. Katopodi, R. S. Meadows, and T. E. Hardingham, "Chondrogenic differentiation of human bone marrow stem cells in transwell cultures: generation of scaffold-free cartilage," Stem Cells, vol. 25, no. 11, pp. 2786-2796, 2007.

[36] S. Hankemeier, M. Keus, and J. Zeichen, "Modulation of proliferation and differentiation of human bone marrow stromal cells by fibroblast growth factor 2: potential implications for tissue engineering of tendons and ligaments," Tissue Engineering Part A, vol. 11, no. 1-2, pp. 41-49, 2005.

[37] L. A. Solchaga, K. Penick, J. D. Porter, V. M. Goldberg, A. I. Caplan, and J. F. Welter, "FGF-2 enhances the mitotic and chondrogenic potentials of human adult bone marrow-derived mesenchymal stem cells," Journal of Cellular Physiology, vol. 203, no. 2, pp. 398-409, 2005.

[38] J. Magalon, O. Bausset, N. Serratrice et al., "Characterization and comparison of 5 platelet-rich plasma preparations in a single-donor model," Arthroscopy: The Journal of Arthroscopic and Related Surgery, vol. 30, no. 5, pp. 629-638, 2014.

[39] R. Zimmermann, R. Jakubietz, M. Jakubietz et al., "Different preparation methods to obtain platelet components as a source of growth factors for local application," Transfusion, vol. 41, no. 10, pp. 1217-1224, 2001.

[40] T. N. Castillo, M. A. Pouliot, and J. L. Dragoo, "Comparison of growth factor and platelet concentration from commercial platelet-rich plasma separation systems," The American Journal of Sports Medicine, vol. 39, no. 2, pp. 266-271, 2011. 


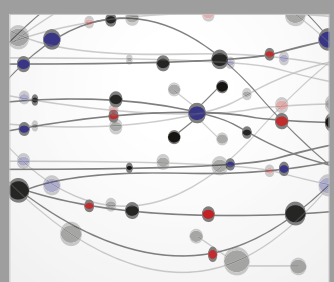

The Scientific World Journal
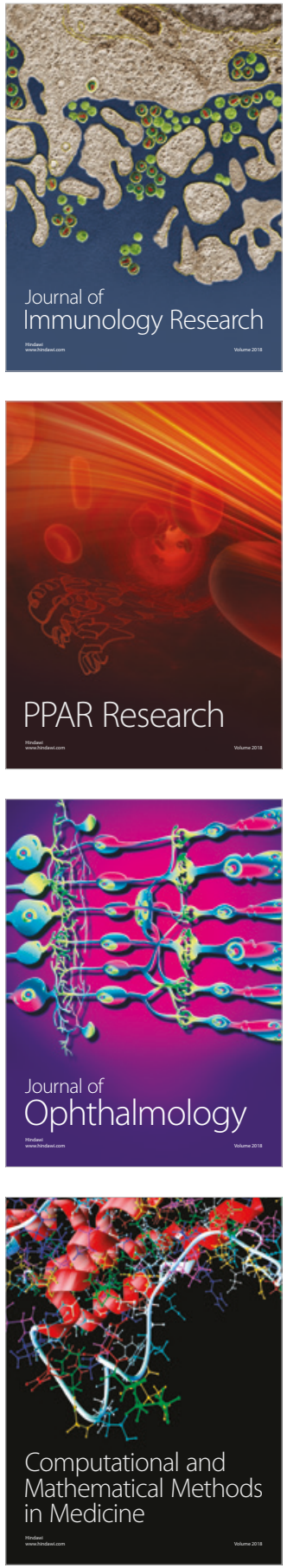

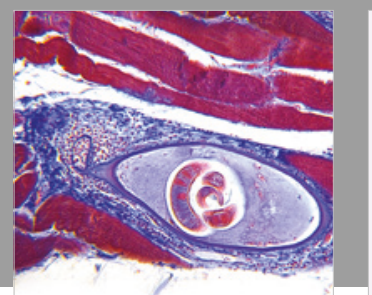

Gastroenterology Research and Practice

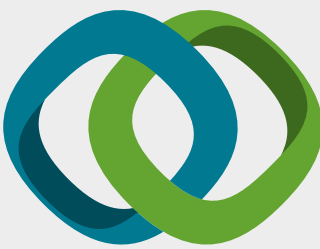

\section{Hindawi}

Submit your manuscripts at

www.hindawi.com
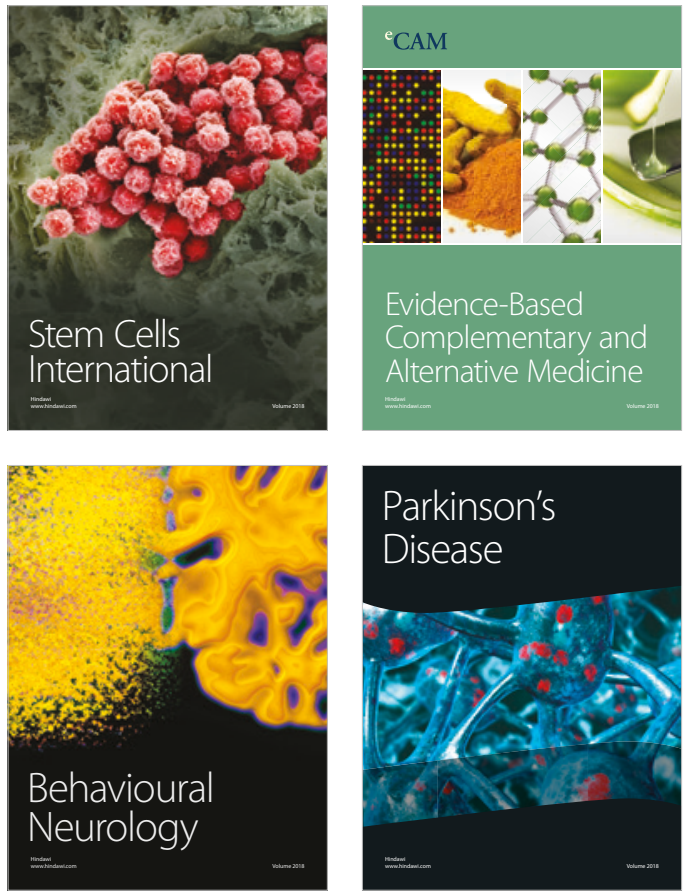

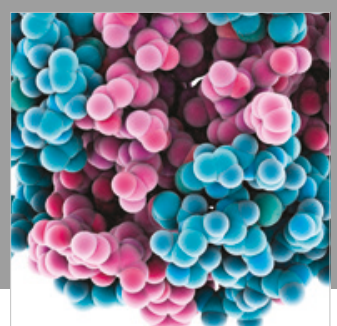

ournal of

Diabetes Research

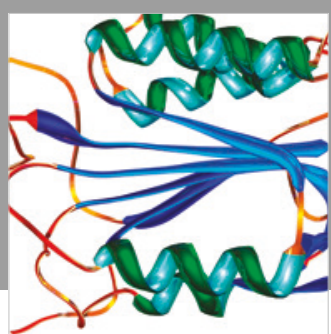

Disease Markers
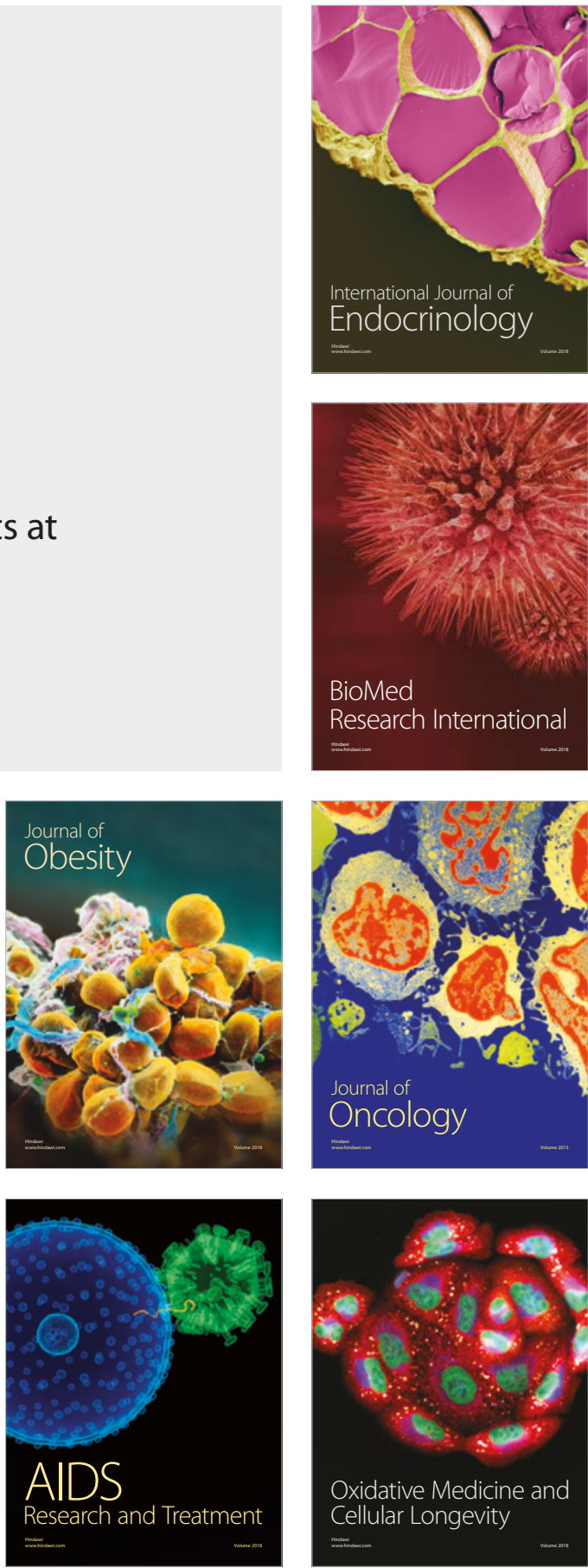